\title{
Distributed Model Predictive Control for Unmanned Aerial Vehicles
}

\author{
Sina Sharif Mansouri ${ }^{1}$, George Nikolakopoulos ${ }^{2}$ and Thomas Gustafsson ${ }^{2}$
}

\begin{abstract}
In this article a distributed model predictive control scheme, for the cooperative motion control of Unmanned Aerial Vehicles (UAVs) is being presented. The UAVs are modeled by a 6-DOF nonlinear kinematic model. Two different control architectures: a centralized and a distributed MPC, are studied and evaluated in simulation experiments. In the centralized approach, one central MPC controller is responsible for the movement coordination of all the UAVs, while in the distributed approach each aerial vehicle plans only for its own actions, while the objective function is coupled with the behavior of the rest of the team members and the constraints are decoupled. In this approach, each agent only shares the future position of itself with the other agents to avoid collisions. For reducing the computation time and complexity, only one step ahead prediction in the corresponding MPC schemes have been considered without a loss of generality. Finally, the efficiency of the overall suggested decentralized MPC scheme, as well as it comparison with the centralized approach, is being evaluated through the utilization of multiple simulation scenarios.
\end{abstract}

\section{IntRoduCtion}

Multi-agent systems are gaining more and more attention since they can be utilized in a wide range of applications, such as search and rescue missions [1], [2], space exploration [3], [4], security patrols [5], [6], traffic control [7], [8], agricultural vehicles [9], garbage collection [10], mapping and land mine removal [11], [12].

For example, the utilization of multiple ground and/or aerials vehicles can be critical during a rescue mission or for example in more complicated missions where a fleet of Unmanned Aerial Vehicles (UAVs) could potentially provide an aerial map for Unmanned Ground Vehicles (UGVs) to complete the rescue mission [13]. In this cases a good cooperation strategy is an important factor to complete the task successfully; as in this case, each agent should fulfill its own sub-task, while sharing sufficient information with the other agents so that the whole system can complete the task. Previous work found in literature

\footnotetext{
${ }^{1}$ Sina Sharif Mansouri is a graduate student of Technical University of Dortmund and he is currently working as a guest researcher at Luleå University of Technology, Department of Computer, Electrical and Space Engineering, Control Engineering Group, Luleå SE-97187, Sweden

${ }^{2}$ George Nikolakopoulos and Thomas Gustafsson are with Luleå University of Technology, Department of Computer, Electrical and Space Engineering, Control Engineering Group, Luleå SE-97187, Sweden
}

is going to be reviewed in Section I-A, have already implemented and simulated UAV controllers in a two dimensional space or have not considered the nonlinear behavior of the UAV dynamical model (or utilized an oversimplified model of unit point dynamics). However, one of the most important contributions in this article is the proposal of a centralized and distributed Model Predictive Control (MPC) scheme for UAVs by utilizing a 6 -DOF full nonlinear model and by considering a free motion in a 3 -Dimensional space.

\section{A. Related Work}

In the area of multi-agent systems, formation control, which consists in controlling multiple agents to achieve their goal and meet their constraints, is one of the most actively studied topics [14]. Various strategies and approaches were studied for solving the motion coordination and formation control problems, which can be classified in three categories as it follows: 1) leaderfollower approach [15], [16], [17], [18], [19], 2) virtual structure approach [20], [21], 3) behavior based approach [22], [23], and [24]. Except from the above strategies, Model Predictive Control (MPC) [25], have been utilized for motion coordination and formation control problems $[26],[27],[28]$ and $[29]$. In these approaches, MPC determined the optimal future control profile according to a prediction of the system behavior over a receding time horizon, while the control actions were computed repetitively by solving a constrained online optimal control problem, over a receding horizon, every time a state measurement or estimate became available. However, one of the drawbacks of the MPC scheme is the fact that it needs an accurate model and its stability becomes one of the main problems, while to guarantee control stability, many approaches have been investigated as those in [30] and $\underline{\text { [31]. }}$.

Furthermore, UAVs' path planning has been widely investigated in the research community, where the main idea is to find the optimum path from the origin to the destination, while avoiding collisions with obstacles and meeting the UAV performance constraints $[32]$. Previous works in this area utilized different methods for solving this issue, for example in [33], the controller was implemented for each UAV to search the environment and identify the maximum number of targets at the end of the search. Their model gain was based on maximizing 
the expected number of targets found. Furthermore, Richards and Jonathan How [34] implemented a Decentralized Model Predictive Control (DMPC) for a team of cooperating UAVs and used coupled constraints to avoid collisions. The above works and many others like the ones in [35], [36], [37], [38], [39], [32] and [40] made one assumption for simplicity or for collision avoidance; that each UAV had a constant altitude (moves on a 2 dimensional plane) or each aerial vehicle moved on different altitudes, an assumption that is restricting the generalisation of the presented approaches.

The 3 dimensional approach, the real flying test case, for UAVs have been however studied in other works on cooperative control for UAVs, like the ones in [41], [42] and [43], where the authors implemented a MPC for the position and heading tracking problem of multiple UAVs in a three-dimensional space, modeled by a lumped parameter approach with 4 degrees of freedom. Their presented MPC scheme presented a better performance when compared to the conventional multi-loop Proportional Derivative (PD) control scheme, while in recent years, several additional research works focused on the implementation of MPC schemes for UAVs in a three dimensional space.

For example, in [44], a distributed linear MPC approach was implemented to solve the trajectory planning problem for rotary wing UAVs, with an objective to form a communication network of multiple targets. In this case, the UAV model was a linear relation between position and velocity, while the attitude of the UAV was not considered and thus the authors did not consider the nonlinear behavior of the UAV. Furthermore, Monteriù et al. in [45], implemented a Nonlinear Decentralized MPC for UAVs focusing in formation based on leaderfollowing approach, where a nonlinear model, based on kinematic equations was obtained describing the motion of a body with six degrees of freedom. Their proposed solution was fully decentralized and was able to take into account the saturation constraints for the control inputs as well as the collision avoidance constraints. Furthermore, in [46], the authors proposed a decentralized MPC and a consensus based control for cooperative formation control strategy for multiple UAVs. In this case and for a flying formation in a three dimensional space, consensus-based control algorithms were applied, while for avoiding collisions, an optimization problem with coupled constraints was solved. Each decoupled UAV was able to create decisions independently and in parallel to other UAVs actions, while the proposed scheme was developed on the linearized model of the quadrotors and their simulation results showed that the proposed control algorithm is effective for formation flying while avoiding collisions.

As mentioned above, there have been many works on cooperative control of UAVs, where most of them implemented and simulated UAV controllers in a two dimensional space as in ([33], [47], [34], [35], [36], [37], [38], [39], [32], [40] and [34]). Few of them utilized a three dimensional space but the authors used a simple linear model or did not consider the attitude problem of the UAV ([44], [46], [48]). In general, only few works considered the nonlinear model in a three dimensional space but still the authors, in this approaches have not considered cooperation strategies and focused only on one UAV control problem ([41], [42], [43] and [45]). Thus, one of the main contributions of this article is the fact that the MPC is implemented for motion control of the team of UAVs that are modelled by nonlinear system dynamics with 6 degrees of freedom. Furthermore, the MPC is utilized for motion control and collision avoidance, while the main advantages of the proposed scheme are: a) the handling of constraints on input and on the states, and b) the utilization of future states, which can be used to avoid collision in the path planning.

In the presented control strategies, two different control architectures are studied, which are: a) the centralized and b) the distributed MPCs. In the case of the centralized MPC, the UAVs are being modelled as an overall system with all the control inputs and actions to be taken under consideration in one overall optimization problem, where the size of the system is mainly dependent on the number of agents and the computation time is increased also by the number of agents and the adopted prediction horizons. In the presented article the centralized system of UAVs is being decomposed into smaller subsystems and a corresponding distributed MPC scheme is proposed, where each subsystem is controlled individually and shares the most recent information with the other agents, while it is assumed that the state variables and constraints are decoupled, but with coupled cost functions.

The remaining of this paper is organized as follow: first the aerial vehicle is modelled in Section II. Different control architectures are studied in Section III, while simulation results that prove the efficiency of the proposed scheme and simulation results are depicted in Sections $\mathrm{IV}$ and $\mathrm{V}$ respectively.

\section{Aerial Vehicle Modeling}

Before developing the motion equation for the UAV, it is important to initially specify the coordinate systems, thus one coordinate system is fixed to the aerial vehicle and is called the body frame, while the other one is called the fixed (or inertial) frame and it is fixed to the Earth (see Fig. 1). The corresponding coordinates are denoted as $x_{f}, y_{f}, z_{f}$ for the fixed frame and $x_{b}, y_{b}, z_{b}$ for the body frame, while $u, v$ and $w$ are the longitudinal, lateral and vertical velocities correspondingly. $P, Q$ and $R$ are the angular velocities of rolling, pitching and yawing respectively. 


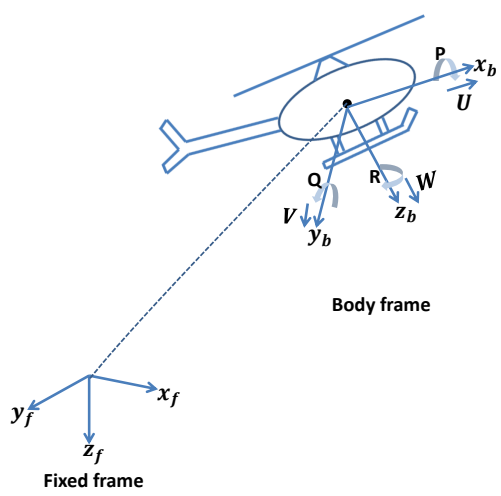

Fig. 1: Body and fixed frames.

The equation for the motion is obtained for the fixed frame [49], while the orientation of the aerial vehicle can be determined by the following three rotations, while prior to applying the following rotation, it is assumed that the aerial vehicle's axis system is in parallel to the fixed frame axis and $x_{f}, y_{f}, z_{f}$ are the axes of the frame:

1) Rotate $x_{f}, y_{f}, z_{f}$ frame through yaw angle $\psi$ to $x_{1}$, $y_{1}, z_{1}$ frame, 2) Rotate $x_{1}, y_{1}, z_{1}$ frame through pitch angle $\theta$ to $x_{2}, y_{2}, z_{2}$ frame, 3) Rotate $x_{2}, y_{2}, z_{2}$ frame through roll angle $\Phi$ to $x_{3}, y_{3}, z_{3}$ frame.

Furthermore, the velocity components can be determined relatively to the fixed frame. By assuming that the velocity along the $x_{f}, y_{f}, z_{f}$ frame is $\frac{d x}{d t}, \frac{d y}{d t}$ and $\frac{d z}{d t}$ respectively, it can be extracted that:

$$
\begin{aligned}
& \frac{d x}{d t}=U_{1} \cos \psi-U_{1} \sin \psi \\
& \frac{d y}{d t}=U_{1} \sin \psi-V_{1} \cos \psi \\
& \frac{d z}{d t}=W_{1}
\end{aligned}
$$

The angular and longitudinal velocities can be expressed as follows:

$$
\begin{aligned}
U_{1} & =U_{2} \cos \theta+W_{2} \sin \theta & U_{2} & =U \\
V_{1} & =V_{2} & V_{2} & =V \cos \theta-W \sin \Phi \\
W_{1} & =-U_{2} \sin \theta+W_{2} \cos \theta & W_{2} & =V \sin \psi+W \cos \psi
\end{aligned}
$$

For reducing the notations, the $\sin \theta, \sin \psi$ and $\cos \psi$ representations are being substituted by $S_{\theta}, S_{\psi}$ and $C_{\psi}$.

From the previous equations' a relation between the linear velocities $\dot{x}, \dot{y}, \dot{z}$ and the $u, v, w$ velocity components can be extracted, where $\dot{x}, \dot{y}, \dot{z}$ are measured in the fixed frame and $U, V$ and $W$ are measured in the body frame.

$$
\left[\begin{array}{c}
\dot{x} \\
\dot{y} \\
\dot{z}
\end{array}\right]=\left[\begin{array}{ccc}
C_{\theta} C_{\psi} & S_{\phi} S_{\theta} C_{\psi}-C_{\phi} S_{\psi} & C_{\Phi} S_{\theta} C_{\psi}+S_{\phi} S_{\psi} \\
C_{\theta} S_{\psi} & S_{\phi} S_{\theta} S_{\psi}+C_{\phi} C_{\psi} & C_{\phi} S_{\theta} S_{\psi}+S_{\phi} C_{\psi} \\
-S_{\theta} & S_{\theta} C_{\theta} & C_{\phi} C_{\theta}
\end{array}\right]\left[\begin{array}{c}
\mathrm{U} \\
\mathrm{V} \\
\mathrm{W}
\end{array}\right]
$$

Also, the relation between the angular velocity in the body frame $(P, Q$, and $R)$ to the Euler rates (derivative of angles) can be obtained as it follows:

$$
\left[\begin{array}{c}
\dot{\phi} \\
\dot{\theta} \\
\dot{\psi}
\end{array}\right]=\left[\begin{array}{ccc}
1 & S_{\phi} \tan _{\theta} & C_{\phi} \tan _{\theta} \\
0 & S_{\phi} C_{\phi} & -S_{\phi} \\
0 & S_{\phi} \sec _{\theta} & C_{\phi} \sec _{\theta}
\end{array}\right]\left[\begin{array}{c}
\mathrm{P} \\
\mathrm{Q} \\
\mathrm{R}
\end{array}\right]
$$

The dynamics of the aerial vehicle can be described by (5) and (6), while the states of the system are represented by the $[x, y, z, \phi, \theta, \psi]^{T}$ vector. The system has 6 inputs $[U, V, W, P, Q, R]^{T}$, where $U, V, W$ are measured in $\mathrm{m} \mathrm{s}^{-1}$ and $P, Q, R$ in $\operatorname{rads}^{-1}$. For more details about the modeling, it is suggested to look at [49], [50] and [51].

\section{Control Design}

The main objective in this article is to control the agents in an unknown environment to reach their goals, deal with actuator limitations, while avoiding collisions and reducing their energy consumption. One advanced controller that can deal with the actual control objectives and operating constraints is Model Predictive Control (MPC). MPC has been popular in practical applications as well as it can be encountered in various control architectures, such as Centralized, Distributed and Decentralized MPCs.

\section{A. Centralized $M P C$}

The classical architecture for the MPC scheme is a centralized control architecture, where one controller is responsible for the whole system as it is depicted in Fig. 2. In general, MPC contains two interacting components: the optimizer and the model. The objective function passed to the optimizer reflects the goal to be attained, and the optimizer uses the model to find the optimal future control sequence without violating the constraints. The way that MPC determines the optimal future control profile is by predicting the system behavior over a receding time horizon. The control actions are computed online by repetitively solving a constrained optimal control problem over a receding horizon every time when a state measurement or estimate becomes available from system. In multi-agent problems, the MPC is responsible for controlling the longitudinal and angular velocities, while satisfying the constraints. In general any

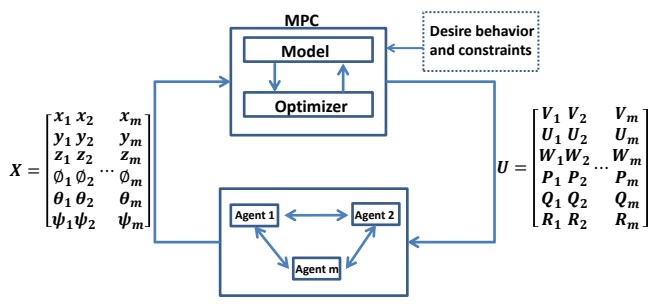

Fig. 2: Centralized MPC.

optimization problem is represented by an objective/cost function that reflects the goals to be attained. Usually it is a minimization (maximization) of some quantity through which different goals can be formulated, such as minimization of energy consumption, maximization of product amount, minimization of raw materials consumption, set-point tracking etc.

At the same time, all real-life processes are operated under some constraints. There can be different reasons 
for these constraints, such as physical limitations or performance specifications. In any case, constraints should be also taken into account and considered in the solution of an optimization problem.

The objective function of this article is defined as the path tracking and corresponding constraints are defined as collision avoidance and actuator limits. In the followings, we assume that we have $m$ agents, where $X_{i, k}=\left(x_{i, k}, y_{i, k}, z_{i, k}, \phi_{i, k}, \theta_{i, k}, \psi_{i, k}\right)$ represent the states of the $i^{\text {th }}$ agent in the $k^{t h}$ horizon, $u_{i}$ is the control vector that is assigned to the $i^{\text {th }}$ agent, $D$ is the minimum accepted distance of each agent and $X_{i}=\left(x_{i}^{d}, y_{i}^{d}, z_{i}^{d}\right)$ is the desired destination of the $i^{\text {th }}$ agent. In this article we are interested in the point mass trajectory of the UAV, while the attitude at which the UAVs may reach at the destination is not taken into account for the objective function. This corresponding problem can then be formulated as:

$$
\begin{aligned}
\text { minimize } & \sum_{k=1}^{N_{p}} \sum_{i=1}^{m} w_{X, i} T_{i}+w_{u, i} \Delta u_{i} \\
\text { subject to } & g(x) \leq 0 \\
& \dot{x}_{i}=f\left(x_{i}, u_{i}\right) \\
& u \in U \\
& x \in X
\end{aligned}
$$

Where $T_{i}$ is the cost function for motion control and is defined as a quadratic cost:

$$
T_{i}=\left(x_{i, k}-x_{i}^{d}\right)^{2}+\left(y_{i, k}-y_{i}^{d}\right)^{2}+\left(z_{i, k}-z_{i}^{d}\right)^{2}
$$

$N_{p}$ is the prediction horizon, with $w_{X, i}$ and $w_{u, i}$ the cost weights, while the presence of the terms with the $\Delta$ operator corresponds to the manipulated variables. These terms are included in order to have influence on the smoothness or aggressiveness of obtained control action. Furthermore, $x \in X$ stands for feasibility of obtained solution and usually used as an additional constraint in the approaches where system model, due to parametrization, is directly included in the optimization routine and $g(x)$ is the constraints of the optimization. In the presented approach, three constraints are defined as it follows:

- Collision Constraints: To avoid collision between the UAVs, the following constraint is imposed.

$$
\left\|X_{i}-X_{j}^{P}\right\|_{2} \geq D \quad i \neq j
$$

The distance of each UAV should not be less than $D$, while the distance between $\mathrm{UAV} i$ and $\mathrm{UAV} j$, with the position of $\left(x_{i}, y_{i}, z_{i}\right)$ and $\left(x_{j}, y_{j}, z_{j}\right)$, is calculated as:

$$
\begin{aligned}
& \left\|X_{i}-X_{j}\right\|_{2}= \\
& \sqrt[2]{\left(x_{i}-x_{j}\right)^{2}+\left(y_{i}-y_{j}\right)^{2}+\left(z_{i}-z_{j}\right)^{2}}
\end{aligned}
$$

The constraint $\left\|X_{i}-X_{j}\right\|_{2} \geq D$ can be defined in a quadratic form as $\left(\left\|X_{i}-X_{j}\right\|_{2}-D\right)^{2}$ and placed in the objective function, but it is necessary to add a weight for this term and tune it. Also it is suggested to assign an "if-condition" so this term is not taken into account in the objective function, as long as the distance is less than $D$ (If the distance is less than $D$ then add $\left(\left\|X_{i}-X_{j}\right\|_{2}-D\right)^{2}$ to the objective function). The collision avoidance term can be calculated for all the prediction horizons but the drawback of this method is the direct increasing of the complexity, the amount of shared information and the computation time. To overcome this complexity, it is assumed that the prediction states of other UAVs are needed only up to a certain stage, while in this article, this constraint must only be satisfied in the first control horizon.

- Velocity Constraints: Assume that the longitudinal and angular velocities have the maximum values of $U_{\max }, V_{\max }, W_{\max }, P_{\max }, Q_{\max }$ and $R_{\max }$. So the constraint on velocity of the $i^{\text {th }}$ agent is defined as:

$$
\left[\begin{array}{c}
-U_{\max } \\
-V_{\max } \\
-W_{\max } \\
-P_{\max } \\
-Q_{\max } \\
-R_{\max }
\end{array}\right] \leq\left[\begin{array}{c}
U_{i} \\
V_{i} \\
W_{i} \\
P_{i} \\
Q_{i} \\
R_{i}
\end{array}\right] \leq\left[\begin{array}{c}
U_{\max } \\
V_{\max } \\
W_{\max } \\
P_{\max } \\
Q_{\max } \\
R_{\max }
\end{array}\right]
$$

- Acceleration Constraints: It should be considered that the rate of the velocity vector is bounded too. Let's assume the velocity vector of the $i^{\text {th }} \mathrm{UAV}$ is called $v_{i}$. So the following constraint for each UAV is defined:

$$
-a \leq \frac{v_{i}(t)-v_{i}(t-1)}{\Delta t} \leq a
$$

Where $\Delta t$ is the time step and $a$ is the maximum allowable rate of change.

The prediction horizon $N_{P}$ stands for the length of the time interval during which the system behavior is predicted; long prediction horizons are required for closed-loop stability and good performance. The length of the prediction horizon is recommended to be chosen in a way that it covers the slowest system mode and the sampling time is recommended to be shorter than the fastest system's mode so that MPC could react timely to disturbances.

The implementation of the MPC as a single agentbased controller generally gives the best performances [52]. In large-scale and complex systems such as power, water distribution, traffic, manufacturing and economic systems, however, undesirable properties (robustness, reliability, scalability, and responsiveness), technical issues (communication delays and computational requirements), commercial, legal, and political issues (unavailability of information and restricted control access) can restrict the usage of a centralized controller. It has been shown that these issues can be solved or at least relieved by breaking the system into sub-systems and using a distributed or a decentralized control scheme [52], where 
local control inputs are computed using local measurements and reduced-order models of the local dynamics. $[53]$.

\section{B. Distributed \& Decentralized MPC}

In Distributed \& Decentralized MPC instead of a single MPC for the overall system, there are multiple MPCs for each subsystem; see Fig. 3 and 4. In multiagent problems, the system can be divided into separate agents with different controllers that may/may not share the information.

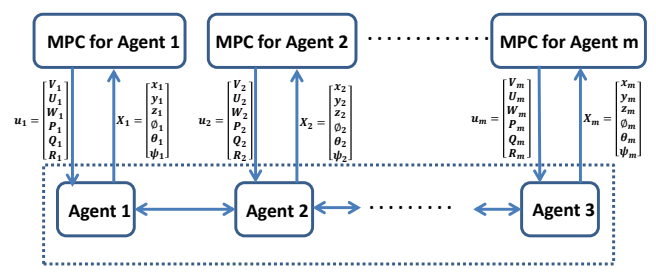

Fig. 3: Decentralized MPC

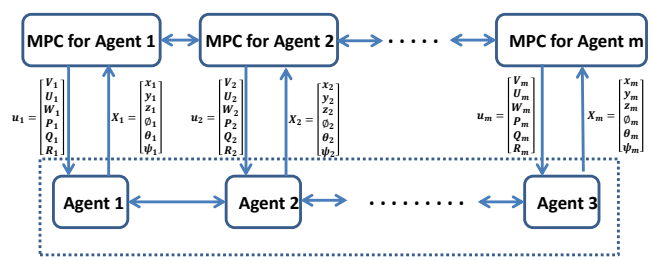

Fig. 4: Distributed MPC

In decentralized MPC each subsystem is controlled independently, without interchanging of information between the subsystems, which can result in a significant loss of information, when the interactions between subsystems are strong or the controllers may be opposing one another's action, for example in multi-agent problems finding the optimal control input without the information of the position of the other agent can cause collisions. In [34], the authors implemented a Decentralized MPC for cooperative UAVs, but also for avoiding collisions, where the agents needed the information of other agents. In their algorithm in each iteration, they solve only once each sub problem respectively and use the information about the latest known intentions of other vehicles. In Figure 3 the arrows between agents denotes the relation between agents, for example sensor or vision information of each agent.

In distributed MPC, on the other hand, each MPC solves a problem by using both local information and the interactions among the systems [52], [53], [54]. The controllers can communicate about the action they would like to perform and share the future states, while reaching to the optimal control inputs. The connection between MPCs in Figure 4 denotes the information that optimizers are shared, as in this case this is the future position of the agents. The Distributed MPC, which is implemented here, has ben inspired by the algorithms in [24], [27] and [55] . For each agent, the optimization problem is:

$$
\begin{aligned}
\text { minimize } & \sum_{k=1}^{N_{p}}\left\{w_{x, i} T_{i}+w_{u, i} \Delta u_{i}\right\}+G_{D} \\
\text { subject to } & g_{i}(x) \leq 0 \\
& \dot{x}_{i}=f\left(x_{i}, u_{i}\right) \\
& u_{i} \in U_{i} \\
& x_{i} \in X_{i}
\end{aligned}
$$

Where $T_{i}$ is the quadratic cost for motion control and is defined the same way as before, while $G_{D}$ is the quadratic cost to avoid collisions and is calculated as:

$$
G_{D}= \begin{cases}w_{i, j}\left(\left\|X_{i}-X_{j}^{P}\right\|_{2}\right)^{2} & \text { distance }<D \\ 0 & \text { otherwise }\end{cases}
$$

In the presented approach, all MPCs for each agent only transfer the future state but do not share their costs. The $X_{j}^{P}$ is the prediction states of the other agents, which remain constant during each optimization. The term $w_{i, j}\left(\left\|X_{i}-X_{j}^{P}\right\|-D\right)^{2}$ is only calculated for the first horizon to reduce complexity and computation time, where $w_{i, j}$ is the weight for collision avoidance and $g_{i}(x)$ is the constraints for the $i^{t h} \mathrm{UAV}$. As the collision avoidance is placed in the objective function, only the velocity and acceleration constraints are considered. In the sequel, it is assumed that $u_{i}^{*}$ and $x_{i}^{*}$ are the optimal control input and state for the agent $i$ respectively. Then the following algorithm could be utilized:

\section{Algorithm:}

Given $u_{i}^{0}, x_{i}(k), 0 \leq w_{u, i}, 0 \leq w_{x, i}, 0 \leq w_{i, j}, 0 \leq w_{i} \leq 1$, $0<P_{\max }$ and $\epsilon>0$

$P \leftarrow 1, K_{i} \leftarrow T, T \ll 1$

While $K_{i}>\epsilon$ and $P \leq P_{\max }$

Do $\forall 1 \leq i \leq M$

Find $u_{i}^{*(P)}$

$u_{i}^{P}=w_{i} u_{i}^{*(P)}+\left(1-w_{i}\right) u_{i}^{P-1}$

Transmit $x_{i}^{*(P)}$ to other agents $i \neq j$.

$K_{i} \leftarrow\left\|u_{i}^{P}-u_{i}^{P-1}\right\|_{\infty}$

end(Do)

end(While)

Where $P_{\max }$ is the maximum number of permissible iterations and it can be called design limitation, $K_{i}$ is the change acceptance of the input and $w_{i}$ is an update parameter.

\section{Simulation Results}

In order to evaluate the Centralized and Distributed $\mathrm{MPC}$, a set of scenarios were considered. For all the scenarios, it was assumed to have the same decoupled dynamics for each UAV. The physical constraints used in these simulation have been gathered from [45], which are used for the quadrotor vehicles. The linear velocities 
were between $-2.5 \mathrm{~m} \mathrm{~s}^{-1}$ and $2.5 \mathrm{~ms}^{-1}$, the angular velocities were between $-0.075 \mathrm{rads}^{-1}$ and $0.075 \mathrm{rads}^{-1}$. Furthermore, the rate of the linear velocity is between $-0.25 \mathrm{~m} \mathrm{~s}^{-2}$ and $0.25 \mathrm{~m} \mathrm{~s}^{-2}$ and the angular acceleration range is from $-0.005 \mathrm{rads}^{-2}$ to $0.005 \mathrm{rad} \mathrm{s}^{-2}$. The controller parameters are shown in Table I. First two to

TABLE I: Control Parameters

\begin{tabular}{|c|c|}
\hline Parameter & Value \\
\hline$w_{x}$ & 10 \\
\hline$w_{y}$ & 10 \\
\hline$w_{z}$ & 10 \\
\hline$w_{u}$ & 1 \\
\hline$w_{i, j}$ & 1000 \\
\hline$N_{p}$ & 3 \\
\hline
\end{tabular}

\begin{tabular}{|c|c|}
\hline Parameter & Value \\
\hline$N_{c}$ & 1 \\
\hline$T_{c}$ & $0.2 \mathrm{~s}$ \\
\hline$K_{i}$ & 1 \\
\hline$P_{\max }$ & 5 \\
\hline$\epsilon$ & 0.1 \\
\hline$D$ & $2 \mathrm{~m}$ \\
\hline
\end{tabular}

five UAVs are controlled by Centralized and Distributed MPCs. Different scenarios are implemented with different origin and destination. Then the computation time and the flight time of each controller are compared. These simulations were tested on a PC with 4 GB RAM and a core $\mathrm{i} 5-3210 \mathrm{M} 2.5 \mathrm{GHz}$. It is assumed that each UAV does not move during the computation of the next control input.

\section{Motion Control}

First two UAVs are controlled. The origin of the first and second UAVs are $[0,0,0]$ and $[20,0,0]$ respectively and the destinations are $[20,20,20]$ and $[0,20,20]$ correspondingly. The simulation results for the two UAVs, which are controlled by Centralized and Distributed MPCs are shown in Figures 5 and 6.

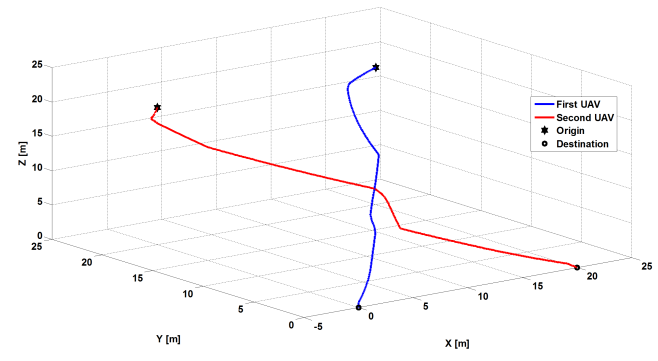

Fig. 5: Centralized MPC for moving two UAVs.

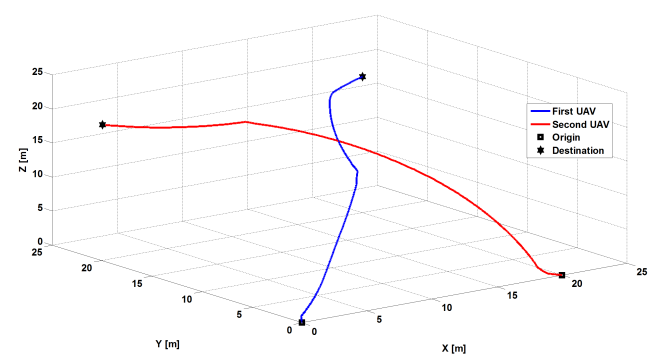

Fig. 6: Distributed MPC for moving two UAVs.

In the sequel, the third UAV start at $[20,20,0]$ and the destination of $[0,0,20]$. In Figures 7 and 9 the trajectory of each agent is shown. In Figures 8 and 10 the distance between three UAVs are shown and as it can be observed in both control scheme the distance was above $2 \mathrm{~m}$.

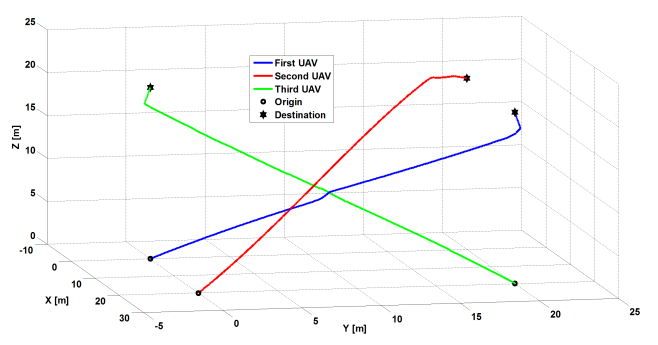

Fig. 7: Centralized MPC for moving three UAVs.

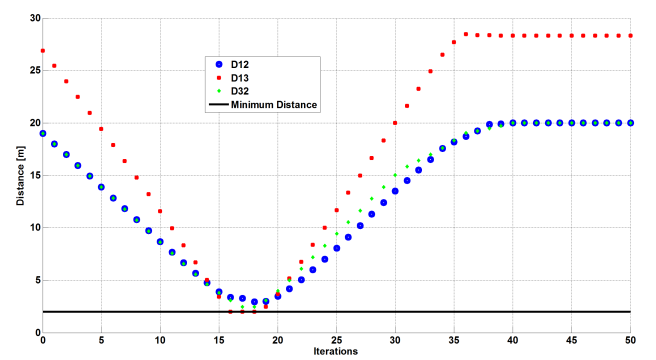

Fig. 8: Distance between three UAVs with Centralized MPC.

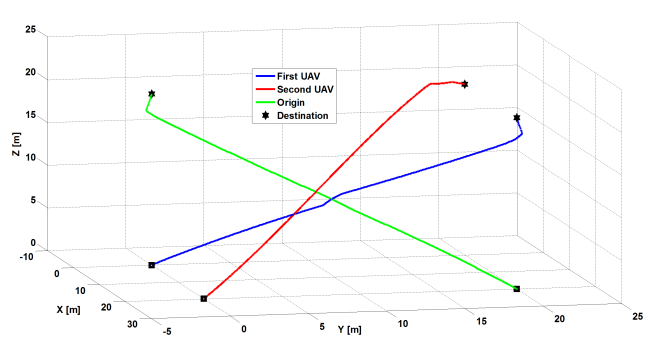

Fig. 9: Distributed MPC for moving three UAVs.

A forth agent has been added with an origin in $[0,20,0]$ and a targeted destination at $[20,20,0]$. In this case the four UAVs were located on the vertices of the square in the $x y$ plane and their paths were at the diagonal of the cube with the volume of $8000 \mathrm{~m}^{3}$. The movement of the agents is being depicted in Figures 11 and 13, while the distance between the UAVs are shown in 12 and 14. The fifth UAV is located in the middle of the $x y$ square with the vertices of the other four UAVs so the origin of it is $[10,10,0]$ and the destination is $[10,10,20]$. The fifth UAV must move vertically to reach its goal. But in this scenario to avoid the collision it is diverted (See Figures 15, 16, 17 and 18). In all above scenarios all UAVs reached to their destination, while avoiding collisions. It can be also observed that each agent instead of following the straight line to destination, diverted to avoid collision. By increasing the number of UAVs, these diversion of the movement is naturally also increasing. The "Fish hooks" behavior of the UAVs path 


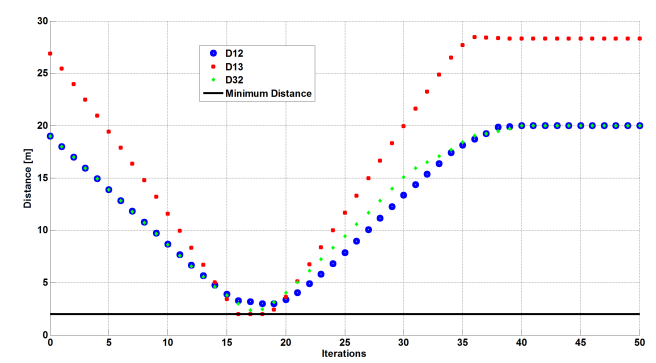

Fig. 10: Distance between three UAVs with Distributed MPC.

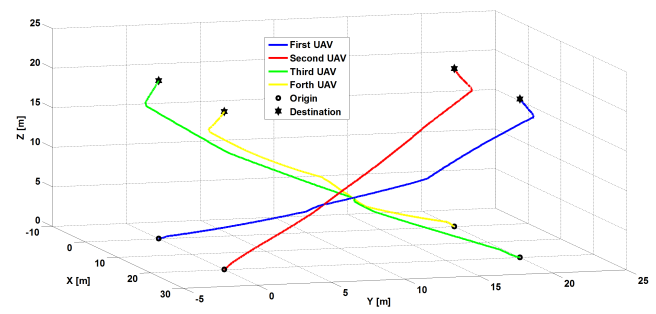

Fig. 11: Centralized MPC for moving four UAVs.

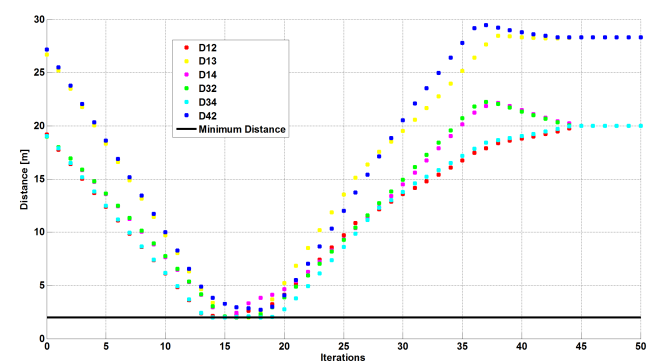

Fig. 12: Distance between four UAVs with Centralized MPC.

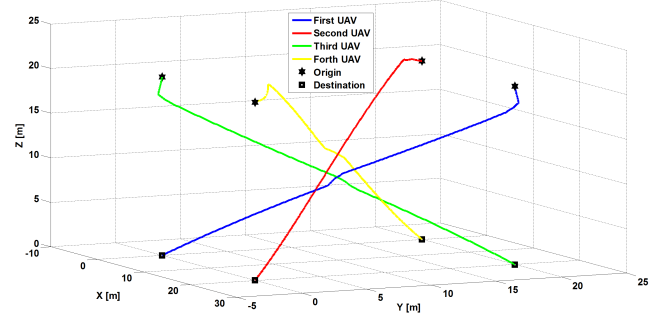

Fig. 13: Distributed MPC for moving four UAVs.

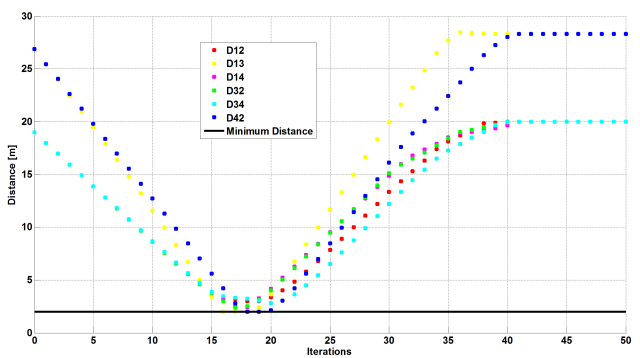

Fig. 14: Distance between four UAVs with Distributed MPC.

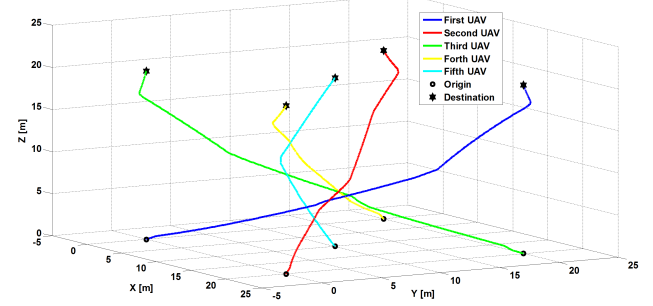

Fig. 15: Centralized MPC for moving five UAVs.

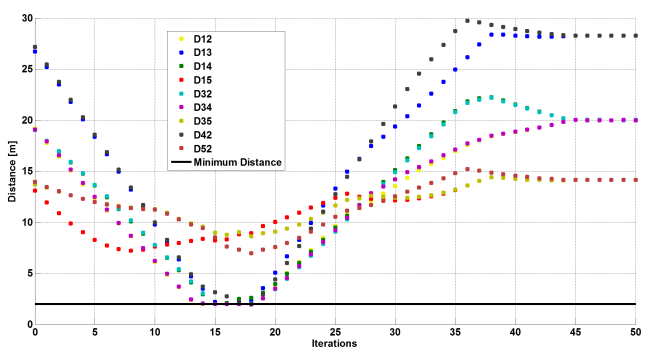

Fig. 16: Distance between five UAVs with Centralized MPC.

can be caused because of: 1) collision avoidance, 2) initial condition for angular position and 3) prediction horizon.

\section{Computation Time}

Another factor, which is important to take in to account, is computation time. The maximum, minimum, average and standard deviation of each controller is provided in Table II. From the obtained results, It can be seen that the computation time of Centralized MPC is increasing by correspondingly the number of UAVs. When the number of agents is two, the difference between two controllers is not considerable, but by increasing the number of agents to more than two, the Distributed MPC offers a considerably less computational time in comparison to the Centralized MPC. For example when the number of agents is 5 , the average computation time for Distributed MPC is 7 times faster than the Centralized MPC. However, it should be considered that $P_{\max }$ and $\epsilon$, the control parameters of Distributed MPC, have a direct relation to the computation time, since the larger $P_{\max }$ and smaller $\epsilon$ is increasing the computation time. In Figure 19, the computation time of each controller

TABLE II: Computation Times

\begin{tabular}{|c|c|c|c|c|}
\hline \multirow{3}{*}{ Number of UAVs } & \multicolumn{5}{|c|}{ Computation Time [s] } \\
\cline { 2 - 5 } & Min & \multicolumn{4}{|c|}{ Mean } & Max & Std. Dev \\
\cline { 2 - 5 } & \multicolumn{4}{|c|}{ Centralized MPC } \\
\hline 2 & 0.42 & 6.8 & 12.42 & 3.53 \\
\cline { 2 - 5 } 3 & .89 & 18.09 & 47.6 & 15.2 \\
\cline { 2 - 5 } 4 & 1.51 & 34.54 & 88.76 & 23.03 \\
\cline { 2 - 5 } 5 & 2.32 & 64.29 & 349.12 & 54.44 \\
\cline { 2 - 5 } & \multicolumn{5}{|c|}{ Distributed MPC } \\
\cline { 2 - 5 } & 0.54 & 3.47 & 9.48 & 1.82 \\
\hline 3 & 1.75 & 6.25 & 16.54 & 3.77 \\
\cline { 2 - 5 } 4 & 1.54 & 7.9 & 21.51 & 4.71 \\
\hline 5 & 2.23 & 9.7 & 35.5 & 7.9 \\
\hline
\end{tabular}




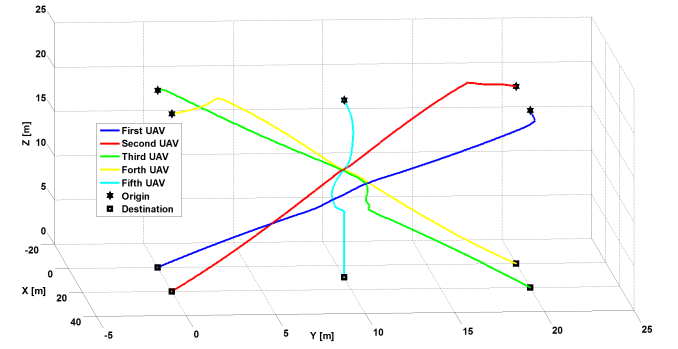

Fig. 17: Distributed MPC for moving five UAVs.

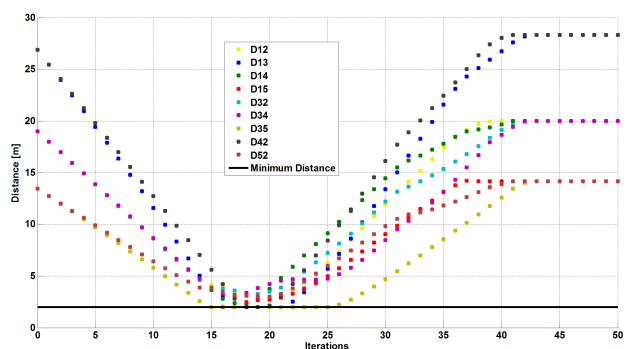

Fig. 18: Distance between five UAVs with Distributed MPC.

is presented. By applying curve fitting, the equation for each computation time is obtained. The centralized MPC is $O\left(n^{2}\right)$ and the distributed MPC is $O(n)$. The $O$ notation is the growth rate of the computation time as the number of UAVs is increased. From the obtained results, it can be directly concluded that the distributed MPC algorithm is more efficient for bigger numbers of UAVs.

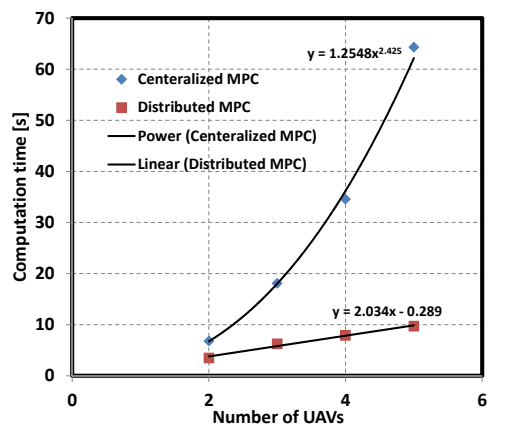

Fig. 19: Computation time mean for Centralized \& Distributed MPCs.

\section{Flight Time}

Finally, in Table III the overall flight time of each proposed controller is being compared. As it can be seen, by increasing the number of UAVs the flight time is also increasing and this was due to the fact that the collision avoidance was active and in general the Centralized MPC managed to have smaller flight time in comparison to Distributed MPC. As it is mentioned before, implementing a MPC scheme as a single agent-based controller generally gives the best performances, however, undesirable properties (robustness, reliability, scalability, and responsiveness), technical issues (communication delays and computational requirements), commercial, legal, and political issues (unavailability of information and restricted control access) are the main restrictions of its usage.

TABLE III: Flight Time Comparison

\begin{tabular}{|c|c|c|}
\hline \multirow{2}{*}{ Number of UAVs } & \multicolumn{2}{|c|}{ Flight Time $[\mathrm{s}]$} \\
\cline { 2 - 3 } & Centralized MPC & Distributed MPC \\
\hline 2 & 8.8 & 8.8 \\
\hline 3 & 8.8 & 9 \\
\hline 4 & 9 & 9.6 \\
\hline 5 & 9.2 & 9.8 \\
\hline
\end{tabular}

\section{Conclusion \& Future Works}

In this paper a Centralized and Distributed MPC scheme has been implemented for cooperative motion control. Centralized and Distributed MPCs determined the optimal future control profile, while the controller satisfied the control objectives and the operating constraints. First, the nonlinear model of the UAV with 6 degrees of freedom has been obtained. Afterwards, the Centralized, Distributed MPCs have been implemented for controlling two of the five UAVs for motion control. In this scenario, the UAVs reached their destination, while avoiding collision with each other. The number of UAVs were increased to increase that a collision avoidance is needed and to compare the performance of the controllers. As it has been indicated, the communication between UAVs was critical to avoid collision. Thus, UAVs shared their future positions with each other. For reducing complexity, only one step ahead prediction of position was shared between the UAVs. From simulations, both control architectures were able to achieve the objective, while guaranteeing the constraint, while the Distributed approach had a significant less computation time when the number of agents is increased to more than 2. Finally, it was shown that the computation time of the centralized MPC was $O\left(n^{2}\right)$ and for the distributed MPC was $O(n)$ so the distributed MPC algorithm was more efficient for more numbers of UAVs. On the other hand Centralized MPC was able to have a little less flight time in comparison to Distributed MPC.

\section{REFERENCES}

[1] P. Doherty and P. Rudol, "A uav search and rescue scenario with human body detection and geolocalization," in AI 200\%: Advances in Artificial Intelligence. Springer, 2007, pp. 1-13.

[2] P. Rudol and P. Doherty, "Human body detection and geolocalization for uav search and rescue missions using color and thermal imagery," in Aerospace Conference, 2008 IEEE. IEEE, 2008, pp. 1-8.

[3] B. Yamauchi, "Frontier-based exploration using multiple robots," in Proceedings of the second international conference on Autonomous agents. ACM, 1998, pp. 47-53.

[4] D. Schoenwald, "Auvs: In space, air, water, and on the ground," IEEE Control Systems Magazine, vol. 20, no. 6, pp. $15-18,2000$. 
[5] A. Machado, G. Ramalho, J.-D. Zucker, and A. Drogoul, "Multi-agent patrolling: An empirical analysis of alternative architectures," in Multi-Agent-Based Simulation II. Springer, 2003, pp. $155-170$.

[6] A. R. Girard, A. S. Howell, and J. K. Hedrick, "Border patrol and surveillance missions using multiple unmanned air vehicles," in Decision and Control, 2004. CDC. 43rd IEEE Conference on, vol. 1. IEEE, 2004, pp. 620-625.

[7] M. Wiering et al., "Multi-agent reinforcement learning for traffic light control," in ICML, 2000, pp. 1151-1158.

[8] B. Burmeister, A. Haddadi, and G. Matylis, "Application of multi-agent systems in traffic and transportation," in Software Engineering. IEE Proceedings-[see also Software, IEE Proceedings/, vol. 144, no. 1 . IET, 1997, pp. 51-60.

[9] L. García-Pérez, M. García-Alegre, A. Ribeiro, and D. Guinea, "An agent of behaviour architecture for unmanned control of a farming vehicle," computers and electronics in agriculture, vol. 60, no. 1, pp. 39-48, 2008.

[10] S. K. Nambiar and S. M. Idicula, "A multi-agent vehicle routing system for garbage collection," in Advanced Computing $(I C o A C), 2013$ Fifth International Conference on. IEEE, 2013, pp. $72-76$.

[11] H. Choset, "Coverage for robotics-a survey of recent results," Annals of mathematics and artificial intelligence, vol. 31, no. 1-4, pp. 113-126, 2001

[12] E. M. Hussein and E. J. Waller, "Landmine detection: the problem and the challenge," Applied Radiation and Isotopes, vol. 53, no. 4, pp. 557-563, 2000.

[13] C. Yao, X. C. Ding, and C. G. Cassandras, "Cooperative receding horizon control for multi-agent rendezvous problems in uncertain environments," in Decision and Control $(C D C)$, 2010 49th IEEE Conference on. IEEE, 2010, pp. 4511-4516.

[14] K.-K. Oh, M.-C. Park, and H.-S. Ahn, "A survey of multiagent formation control," Automatica, vol. 53, pp. 424-440, 2015.

[15] A. K. Das, R. Fierro, V. Kumar, J. P. Ostrowski, J. Spletzer, and C. J. Taylor, "A vision-based formation control framework," Robotics and Automation, IEEE Transactions on, vol. 18, no. 5, pp. 813-825, 2002.

[16] M. B. Egerstedt and X. Hu, "Formation constrained multiagent control," 2001.

[17] N. E. Leonard and E. Fiorelli, "Virtual leaders, artificial potentials and coordinated control of groups," in Decision and Control, 2001. Proceedings of the 40th IEEE Conference on, vol. 3. IEEE, 2001, pp. 2968-2973.

18] B. Anderson, C. Yu, B. Fidan, and J. M. Hendrickx, "Rigid graph control architectures for autonomous formations," Control Systems, IEEE, vol. 28, no. 6, pp. 48-63, 2008.

[19] A. N. Bishop, T. H. Summers, and B. D. Anderson, "Control of triangle formations with a mix of angle and distance constraints," in Control Applications (CCA), 2012 IEEE International Conference on. IEEE, 2012, pp. 825-830.

[20] W. Ren and R. Beard, "Decentralized scheme for spacecraft formation flying via the virtual structure approach," Journal of Guidance, Control, and Dynamics, vol. 27, no. 1, pp. 73-82, 2004.

[21] M. A. Lewis and K.-H. Tan, "High precision formation control of mobile robots using virtual structures," Autonomous Robots, vol. 4, no. 4, pp. 387-403, 1997.

[22] T. Balch and R. C. Arkin, "Behavior-based formation control for multirobot teams," Robotics and Automation, IEEE Transactions on, vol. 14, no. 6, pp. 926-939, 1998.

[23] C. R. McInnes, "Autonomous ring formation for a planar constellation of satellites," Journal of Guidance, Control, and Dynamics, vol. 18, no. 5, pp. 1215-1217, 1995.

[24] K. Kanjanawanishkul and A. Zell, "Distributed model predictive control for coordinated path following control of omnidirectional mobile robots," in Systems, Man and Cybernetics, 2008. SMC 2008. IEEE International Conference on. IEEE, 2008, pp. 3120-3125.

[25] E. F. Camacho and C. B. Alba, Model predictive control. Springer Science \& Business Media, 2013.
[26] W. B. Dunbar and R. M. Murray, "Distributed receding horizon control for multi-vehicle formation stabilization," Automatica, vol. 42, no. 4, pp. 549-558, 2006.

[27] R. L. Raffard, C. J. Tomlin, and S. P. Boyd, "Distributed optimization for cooperative agents: Application to formation flight," in Decision and Control, 2004. CDC. 43rd IEEE Conference on, vol. 3. IEEE, 2004, pp. 2453-2459.

[28] E. Franco, T. Parisini, and M. M. Polycarpou, "Cooperative control of distributed agents with nonlinear dynamics and delayed information exchange: a stabilizing receding-horizon approach," in Decision and Control, 2005 and 2005 European Control Conference. CDC-ECC'05. 44th IEEE Conference on. IEEE, 2005, pp. 2206-2211.

[29] K. Hashimoto, S. Adachi, and D. V. Dimarogonas, "Distributed aperiodic model predictive control for multi-agent systems," Control Theory \& Applications, IET, vol. 9, no. 1, pp. 10-20, 2015.

[30] F. Allgower, R. Findeisen, Z. K. Nagy et al., "Nonlinear model predictive control: from theory to application," Journal of the Chinese Institute of Chemical Engineers, vol. 35, no. 3, pp. 299-315, 2004.

[31] D. Q. Mayne, J. B. Rawlings, C. V. Rao, and P. O. Scokaert, "Constrained model predictive control: Stability and optimality," Automatica, vol. 36, no. 6, pp. 789-814, 2000

[32] M. Yao and M. Zhao, "Unmanned aerial vehicle dynamic path planning in an uncertain environment," Robotica, vol. 33 , no. 03, pp. 611-621, 2015.

[33] M. Flint, M. Polycarpou, and E. Fernandez-Gaucherand, "Cooperative control for multiple autonomous uav's searching for targets," in Decision and Control, 2002, Proceedings of the 41st IEEE Conference on, vol. 3. IEEE, 2002, pp. 2823-2828.

[34] A. Richards and J. How, "Decentralized model predictive control of cooperating uavs," in Decision and Control, 2004. CDC. 43rd IEEE Conference on, vol. 4. IEEE, 2004, pp. 4286-4291.

[35] D. M. Stipanović, G. Inalhan, R. Teo, and C. J. Tomlin, "Decentralized overlapping control of a formation of unmanned aerial vehicles," Automatica, vol. 40, no. 8, pp. 1285-1296, 2004.

[36] J. How, E. King, and Y. Kuwata, "Flight demonstrations of cooperative control for uav teams," in AIAA 3rd unmanned unlimited technical conference, workshop and exhibit, 2004.

[37] R. W. Beard and T. W. McLain, "Multiple uav cooperative search under collision avoidance and limited range communication constraints," in Decision and Control, 2003. Proceedings. 42nd IEEE Conference on, vol. 1. IEEE, 2003, pp. 25-30.

[38] E. P. Anderson, R. W. Beard, and T. W. McLain, "Real-time dynamic trajectory smoothing for unmanned air vehicles," Control Systems Technology, IEEE Transactions on, vol. 13, no. 3, pp. 471-477, 2005.

[39] A. Ryan, J. Tisdale, M. Godwin, D. Coatta, D. Nguyen, S. Spry, R. Sengupta, and J. K. Hedrick, "Decentralized control of unmanned aerial vehicle collaborative sensing missions," in American Control Conference, 2007. ACC'07. IEEE, 2007, pp. 4672-4677.

[40] H. Peng, M.-l. Huo, Z.-z. Liu, and W. Xu, "Simulation analysis of cooperative target search strategies for multiple uavs," in Control and Decision Conference (CCDC), 2015 27th Chinese, May 2015, pp. 4855-4859.

[41] H. J. Kim, D. H. Shim, and S. Sastry, "Nonlinear model predictive tracking control for rotorcraft-based unmanned aerial vehicles," in American Control Conference, 2002. Proceedings of the 2002, vol. 5. IEEE, 2002, pp. 3576-3581.

[42] H. J. Kim and D. H. Shim, "A flight control system for aerial robots: algorithms and experiments," Control engineering practice, vol. 11, no. 12, pp. 1389-1400, 2003.

[43] D. H. Shim, H. J. Kim, and S. Sastry, "Decentralized nonlinear model predictive control of multiple flying robots," in Decision and control, 2003. Proceedings. 42nd IEEE conference on, vol. 4. IEEE, 2003, pp. 3621-3626.

[44] A. Grancharova, E. I. Grøtli, and T. A. Johansen, "Uavs trajectory planning by distributed mpc under radio communi- 
cation path loss constraints," Journal of Intelligent $\&$ Robotic Systems, pp. 115-134, 2014.

[45] A. Monteriù, "Nonlinear decentralized model predictive control for unmanned vehicles moving in formation," Information Technology And Control, vol. 44, no. 1, pp. 89-97, 2015.

[46] Y. KURIKI and T. NAMERIKAWA, "Formation control with collision avoidance for a multi-uav system using decentralized mpc and consensus-based control," SICE Journal of Control, Measurement, and System Integration, vol. 8, no. 4, pp. 285$294,2015$.

[47] R. W. Beard, T. W. McLain, M. Goodrich, E. P. Anderson et al., "Coordinated target assignment and intercept for unmanned air vehicles," Robotics and Automation, IEEE Transactions on, vol. 18, no. 6, pp. 911-922, 2002.

[48] K. Alexis, C. Papachristos, R. Siegwart, and A. Tzes, "Robust model predictive flight control of unmanned rotorcrafts," Journal of Intelligent $\&$ Robotic Systems, pp. 1-27, 2015.

[49] R. C. Nelson, Flight stability and automatic control. WCB/McGraw Hill, 1998, vol. 2.

[50] B. Etkin and L. Reid, Dynamics of Flight: Stability and Control. Wiley, 1995. [Online]. Available: https://books.google.de/books?id=ET65QgAACAAJ

[51] C. Perkins, Airplane Performance Stability and Control. Central Book Company, 1970. [Online]. Available: https://books.google.de/books?id=5W3hoAEACAAJ

[52] R. Negenborn and H. Hellendoorn, "Intelligence in transportation infrastructures via model-based predictive control," in Intelligent Infrastructures. Springer, 2010, pp. 3-24.

[53] E. Camponogara, D. Jia, B. H. Krogh, and S. Talukdar, "Distributed model predictive control," Control Systems, IEEE, vol. 22 , no. 1, pp. 44-52, 2002.

[54] R. Negenborn and J. Maestre, "Distributed model predictive control: An overview and roadmap of future research opportunities," Control Systems, IEEE, vol. 34, no. 4, pp. 87-97, 2014.

[55] A. N. Venkat, "Distributed model predictive control: theory and applications," Ph.D. dissertation, UNIVERSITY OF WISCONSIN-MADISON, 2006. 